SS/Fibro-group were more likely reporting arthralgia symptoms (100.0\%) than SS-group (76.0\% $\mathrm{p}=0.02)$, despite similar clinical evidence of arthritis-synovitis among the two groups $(12.0 \%$ in both groups respectively, $p=1.00)$. Moreover, SS/Fibro-group showed significantly lower ESSDAI score $(2.8 \pm 1.7)$ and higher ESSPRI score $(7.0 \pm 0.9)$ compared to SS-group (ESSDAI: $7.5 \pm 3.7 p<0.001$ and ESSPRI: $5.2 \pm 1.4, \mathrm{p}<0.001$ respectively). Finally, analyzing the differential distribution of individual scores of physical and psycological domains of the Italian-FIQR Questionnaire, SS/Fibro-group did not differ compared to Fibro-group ( $p>0.05$ for all the 21 questions included).

Conclusion: SS is affected by concomitant fibromyalgia in terms of subjective-dependent parameters (i.e. joint complaints) however the concomitant SS does not affect the impact of fibromyalgia on physical and psycological domains, even if disease activity is higher in SS patients without fibromyalgia.

References:

[1] Shiboski SC et al. Arthritis Care Res, 2012

[2] Wolf F. et al. Arthritis Rheum 1990

Disclosure of Interests: Annunziata Capacci: None declared, Pietro Rubortone: None declared, Valentina Varriano: None declared, Annamaria Paglionico: None declared, Simone Perniola: None declared, Maria Rita Gigante: None declared, Barbara Tolusso: None declared, Stefano Alivernini: None declared, Elisa Gremese Speakers bureau: Abbvie, BMS, Celgene, Jannsen, Lilly, MSD, Novartis, Pfizer, Sandoz, UCB

DOI: 10.1136/annrheumdis-2020-eular.5940

\section{AB0948 OF SYMPTOMS IN PATIENTS PRESENTING TO EARLY ARTHRITIS CLINICS: A RETROSPECTIVE ANALYSIS OF 279 PATIENTS}

S. Dar ${ }^{1}$, D. Ludwig ${ }^{2}$, M. Greenwood ${ }^{2}$, H. Kazkaz ${ }^{2}$, V. Morris ${ }^{2} .{ }^{1}$ UCL Medical School, London, United Kingdom; ${ }^{2}$ University College Hospital, London, United Kingdom

Background: Joint hypermobility is a common, although largely ignored physical sign. It is often asymptomatic but can be associated with significant musculoskeletal symptoms. Joint hypermobility may also be a feature of an underlying genetic disorder and patients may present with arthralgia, recurrent soft tissue injuries and intermittent joint swelling due to mechanical instability and poor proprioception. At University College London Hospital, we run a national service for the diagnosis and management of patients with hypermobility related disorders including hypermobility spectrum disorders, Ehlers-Danlos syndromes and Marfan syndrome. Over the years we observed that a significant number of our patients had been referred to the early arthritis clinics years prior to the recognition of their hypermobility. For example, one patient with a vascular type of Ehlers-Danlos syndrome EDS (confirmed COL3A mutation) presented to 3 different hospitals over a 5-year period, with possible inflammatory arthritis prior to the EDS diagnosis. Several studies have shown that a significant proportion of patients attending early arthritis clinics do not have inflammatory rheumatic diseases. In our experience, heritable disorders of connective tissue and hypermobility spectrum disorders are often overlooked and should be included in the differential diagnosis in patients seen in the early arthritis clinics.

Objectives: We aimed to audit the outcome of patients who were seen in the early arthritis clinics focusing on those who were not found to have inflammatory rheumatic diseases and to explore if joint hypermobility was considered as a possible cause of patient's symptoms.

Methods: A retrospective analysis of medical records was conducted of patients attending the early arthritis clinics at University College London Hospital between May 2018 and December 2019.

Results: 279 patients (90 males, 189 females) were seen in the early arthritis clinics with a mean age of 48 (range 19-91). 131 patients (47\%) did not have inflammatory rheumatic diseases. Sixty-three of these patients $(48 \%)$ were not given any diagnosis and joint hypermobility was not assessed during the appointment. Eleven patients $(8 \%)$ had features of hypermobility, 11 patients $(8 \%)$ were diagnosed with fibromyalgia, 20 patients (15\%) received a diagnosis of osteoarthritis, and 27 patients $(21 \%)$ were given other diagnoses including tendonitis and soft tissue pathology.

Conclusion: Almost $50 \%$ of patients who were seen in the early arthritis clinics did not have inflammatory rheumatic diseases and $21 \%$ of patients were discharged without a clear diagnosis. In these patients, hypermobility was not assessed and this is consistent with our observation. In our experience recognising joint hypermobility as a cause of arthralgia and intermittent joint swelling usually reassures patients and motivates them to follow appropriate treatment protocols including physiotherapy and occupational therapy thus allowing a more efficient utilization of early arthritis clinic resources towards those with true inflammatory rheumatic diseases. Going forward, we have planned to embed a cognisant attitude towards hypermobility within the relevant clinics to ensure that patients who do not have inflammatory arthritis are assessed for hypermobility and directed towards appropriate management.

References:

[1] Castori M, Hakim A. Contemporary approach to joint hypermobility \& related disorders. Current Opinion in Pediatrics. 2017;29(6):640-649.

[2] Emma Connelly, Alan Hakim, Sally Davenportc \& Jane Simmonds, A study exploring the prevalence of Joint Hypermobility Syndrome in patients attending a Musculoskeletal Triage Clinic. Physiotherapy Practice \& Research 36 (2015) 43-53

[3] Feuchtenberger M, Nigg A, Kraus M, Schäfer A. Rate of Proven Rheumatic Diseases in a Large Collective of Referrals to an Outpatient Rheumatology Clinic under Routine Conditions. Clin Med Insights Arthritis Musculoskelet Disord. 2016; 9: 181-187.

Disclosure of Interests: None declared

DOI: 10.1136/annrheumdis-2020-eular.4548

\section{AB0949 \\ AUTONOMIC AND INFLAMMATORY MECHANISMS OF PAIN AND FATIGUE IN FIBROMYALGIA AND ME/CFS: AN INTERVENTIONAL STUDY}

J. Eccles $^{1,2}$, M. Amato ${ }^{1}$, C. Thompson ${ }^{1,2}$, K. Themelis ${ }^{1,3}$, H. Critchley ${ }^{1}$,

N. Harrison ${ }^{1,4}$, K. Davies ${ }^{1,2}$. 'Brighton and Sussex Medical School, Falmer, United Kingdom; ' ${ }^{2}$ Clinical Investigation and Research Unit, Royal Sussex County Hospital, Brighton, United Kingdom; ${ }^{3}$ University of Warwick, Warwick, United Kingdom; ${ }^{4}$ CUBRIC, University of Cardiff, Cardiff, United Kingdom

Background: Fibromyalgia and ME/CFS are complex disorders with overlapping symptoms; the pathoaetiology and clinical distinction are debated, however inflammatory and autonomic abnormalities are observed.

Objectives: To investigate the role of inflammatory and autonomic nervous system responses in mechanisms of pain and fatigue in fibromyalgia and ME/CFS

Methods: 63 patient participants with clinical diagnoses of fibromyalgia and/or ME/CFS were recruited into a multi-stage interventional study (ISRCTN78820481) alongside 24 healthy controls. All underwent research diagnostic criteria evaluation. The majority underwent autonomic challenge (60 degree head up tilt) and/ or inflammatory challenge (placebo-controlled typhoid vaccination) with baseline characterisation of symptoms, inflammatory markers and pre-post measures of pain and fatigue.

Results: Of the 63 patients, $32 \%$ of patients had received a clinical diagnosis of Fibromyalgia; 38\% ME/CFS and 30\% dual diagnoses. Following research evaluation $89 \%$ met ACR diagnostic criteria for fibromyalgia; 94\% Canadian Criteria for ME/CFS; $97 \%$ Fukada Criteria for ME/CFS

There was a significantly higher ESR in patients compared to controls $(p=0.036)$ There was a trend towards higher CRP in patients compared to controls $(p=0.076)$ ESR correlated with baseline pain score $(r=0.309, p=0.011)$, fatigue severity $(r=0.262, p=0.032)$, fatigue impact $(r=0.382, p=0.014)$ change in fatigue score induced by tilt $(r=0.319, p=0.011)$ and change in pain score induced by placebo-controlled inflammation $(r=-0.279, p=0.043)$. Similarly CRP level correlated with baseline pain score $(r=0.340, p=0.005)$, fatigue impact $(r=0.439, p=0.004)$, change in fatigue $(r=-0.277, p=0.045)$ and pain score $(r=-0.394, p=0.014)$ induced by placebo-controlled inflammation and change in pressure pain theshold induced by tilt $(r=0.286, p=0.027)$.

Baseline IL6 was higher in patients than controls $(p=0.002)$, correlating with baseline pain score $(r=0.345, p=0.002)$ and change in pain score induced by tilt $(r=0.281, p=0.21)$. Change in IL6 induced by inflammatory challenge correlated with inflammation induced fatigue score $(r=0.378, p=0.01)$

Conclusion: Inflammatory and autonomic mechanisms contribute to pain and fatigue in this frequently overlooked patient group, highlighting possibilities for targeted treatments. Such data will be enriched going forward by neuroimaging and transcriptomic insights.

References: $n / a$

Acknowledgments: This work was supported by Versus Arthritis, Action for Me, Fibroduck Foundation and NIHR

Disclosure of Interests: Jessica Eccles: None declared, Marisa Amato: None declared, Charlotte Thompson: None declared, Kristy Themelis: None declared Hugo Critchley: None declared, Neil Harrison Grant/research support from: Yes, Speakers bureau: Yes, Kevin Davies: None declared DOI: 10.1136/annrheumdis-2020-eular.5607

\begin{tabular}{l|l}
\hline AB0950 & EFFECT OF SELECTIVE SEROTONIN REUPTAKE \\
INHIBITORS AND SEROTONIN NOREPINEPHRINE \\
REUPTAKE INHIBITORS ON BONE MINERAL \\
DENSITY IN EGYPTION PATIENTS WITH PRIMARY \\
FIBROMYALGIA
\end{tabular}

Y. Gazar ${ }^{1}$ on behalf of prof dr. Hesham salah HamoudDr. Mohammed Magdy GhaitDr. Mohammed Harb. ${ }^{1}$ Rheumatology Department, Faculty of Medicine, AL-Azhar University, Cairo, Egypt 